\title{
NUMERICAL APPROXIMATIONS CAN CREATE \\ CHAOS-RELATED ERRORS IN BLOCKING FORECASTS
}

\section{Rinne, Juhani}

1992-05

Rinne , J \& Järvinen , H 1992 , ' NUMERICAL APPROXIMATIONS CAN CREATE

CHAOS-RELATED ERRORS IN BLOCKING FORECASTS ' , Monthly weather review , vol.

120 , no. 5 , pp. 884-889 . https://doi.org/10.1175/1520-0493(1992)120<0884:NACCCR>2.0.CO;2

http://hdl.handle.net/10138/313516

https://doi.org/10.1175/1520-0493(1992)120<0884:NACCCR>2.0.CO;2

publishedVersion

Downloaded from Helda, University of Helsinki institutional repository.

This is an electronic reprint of the original article.

This reprint may differ from the original in pagination and typographic detail.

Please cite the original version. 


\title{
Numerical Approximations Can Create Chaos-related Errors in Blocking Forecasts
}

\author{
JUHANI RINNE AND HEIKKI JÄRVINEN \\ Department of Meteorology, University of Helsinki, Helsinki, Finland
}

(Manuscript received 26 December 1990, in final form 3 July 1991)

ABSTRACT

\begin{abstract}
The chaotic structure of two-dimensional atmospheric flow is illustrated. It is shown that certain errors in numerical approximations can prevent the correct prediction of chaotic processes. This is the case when the numerical approximations do not sufficiently allow air parcels to deviate from each other. The error mechanism is described with a case study and is proposed as one explanation for the errors observed when forecasting the development of blocking highs. It can explain why the errors in blocking highs are similarly found in different models from different centers, why they appear in medium-range forecasts but not in short-range forecasts, and why the error decreases only slowly with increasing resolution.
\end{abstract}

\section{Introduction}

The generation and development of blocking highs in numerical forecasts is often seriously wrong. The reasons are not well known (CAS/JSC Working Group on Numerical Experimentation 1988; Tibaldi and Molteni 1990). The error mechanism must be quite universal because blocking highs are similarly problematic in different models from different forecasting centers.

We illustrate, with a case study, that a procedure as simple as averaging, when applied in the models' numerical approximations, can lead to erroneous vorticity feeding in blocking areas and can cause the erroneous development of highs. The mechanism is typical of a chaotic stream and is not limited to only atmospheric applications. It is caused by those numerical approximations that do not allow air parcels to deviate enough from each other, thus partly preventing the chaotic processes.

We see blocking highs as features of a chaotic flow, a view that has stimulated our special interest in them. In the present results, air parcels reaching a ridge disperse quite differently from those avoiding the ridge.

The forecast model used is a simple two-dimensional Lagrangian parcel-advection model with specified winds. A modification in the numerical approximations used leads to essential changes in the properties of air parcels flowing into a ridge of high pressure. We first describe the control case and then modify it to produce the disturbed case.

Corresponding author address: Heikki Järvinen, University of Helsinki, Department of Meteorology, Hallituskatu 11-13, Finland.

\section{The data and the model}

The problem considered here is how to compute the trajectory of an air parcel when the wind field is given at every moment. Formally, this problem is a forecasting one because the trajectory depends on time and is determined from

$$
\frac{d \mathbf{s}}{d t}=\mathbf{v},
$$

where $|\mathbf{s}|$ gives the length of the trajectory, and $|\mathbf{v}|$ is the speed of the wind. Equation (1) is integrated by applying a semi-implicit time-stepping scheme as follows:

$$
\begin{aligned}
& \mathbf{s}_{n+1}^{\prime}=\mathbf{s}_{n}+\Delta t \mathbf{v}\left(\mathrm{s}_{n}\right), \\
& \mathbf{s}_{n+1}=\mathbf{s}_{n}+\left(\frac{\Delta t}{2}\right)\left[\mathbf{v}\left(\mathrm{s}_{n}\right)+\mathrm{v}\left(\mathrm{s}_{n+1}^{\prime}\right)\right] .
\end{aligned}
$$

Thus, these studies are restricted to the quasi-horizontal case; that is, vertical velocities are omitted. The length of the time step $\Delta t$ and the grid interval $\Delta x$ are $1 \mathrm{~h}$ and $381 \mathrm{~km}$ (at $60^{\circ} \mathrm{N}$ on a stereographic projection), respectively. The $500-\mathrm{hPa}$ height analyses were obtained from Fleet Numerical Weather Central (FNWC) and given on the National Meteorological Center (NMC) grid at a total of 1404 points north of $20^{\circ} \mathrm{N}$.

The value of the wind at the actual point is interpolated linearly from the grid winds, which in turn have been geostrophically determined from the height values given at the grid points. Therefore, the interpolation in space is linear. The formulas applied are explained in the Appendix. 
The interpolation in time is made with a forecasting model and is, thus, fully nonlinear. The height fields are given only at the main observation times, 0000 and 1200 UTC. Interpolation between these times is made by predicting with a barotropic model forward and backward from the initial conditions at 0000 and 1200 UTC, respectively. A weighted average of these two forecasts is computed so that the closer the actual time is to 0000 UTC ( 1200 UTC) the more weight is given to the forward (backward) forecast. As a result, a series of height fields having a single time-step interval is obtained. The series begins and ends with FNWC analyses. A similar procedure is applied for the $12-\mathrm{h}$ interval beginning with the 1200 UTC analysis.

\section{The control case}

Three different groups of freely traveling parcels were released at 0000 UTC 4 March 1965 and then followed for 6 days, applying (2) to each parcel. Each group consisted of 2601 air parcels, which were initially dis- tributed uniformly within squares of $0.1^{\circ} \times 0.1^{\circ}$. The groups were separated by $1^{\circ}$ in a north-south direction. The movement of the three groups $[(a)-(c)]$ is shown in Fig. 1.

In case (c), the points are still, after 6 days of travel, so close to each other that only one spot can be seen in Fig. 1. In case (a), the parcels have deviated only slightly so that they still form rather a compact group. The behavior of the middle group, (b), illustrates the chaotic nature of atmospheric flow. Although this group was initially very close to the other groups-in fact right between them-it has now spread unexpectedly over a wide area. Note that some parcels are now in the area of a ridge (indicated by $\mathrm{H}$ ). This ridge never developed into a blocking high, but it can be taken as an illustrative candidate for one.

Figure 1 also clearly demonstrates how sensitive the trajectory computations can be to the initial conditions. A small error in either the location of a parcel or the analyzed wind field can lead to quite a different final location for the parcel. Consider, for instance, the case of Chernobyl. Of course the result of this study is

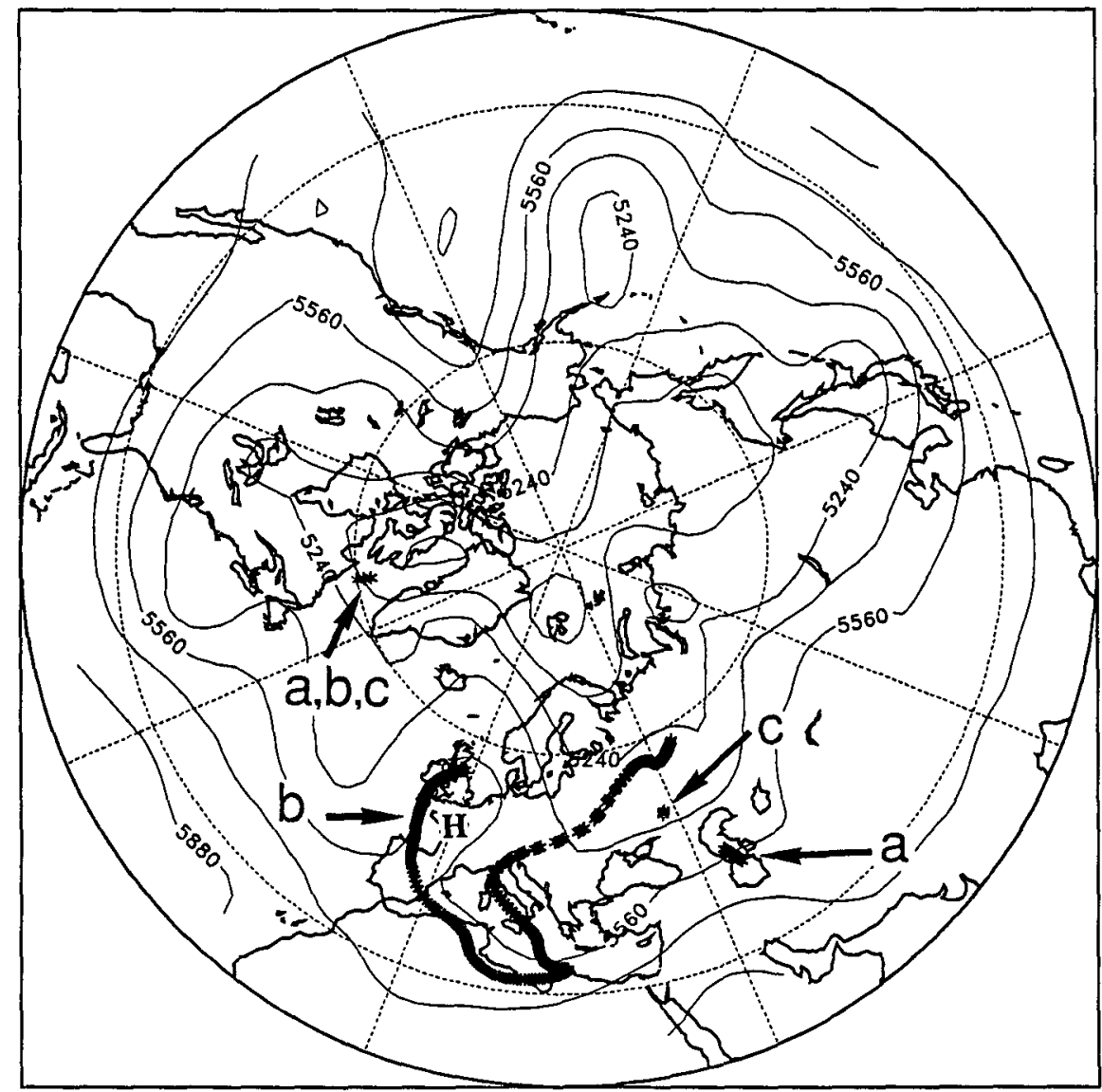

FIG. 1. The movement of three groups of air parcels $(*)$, initially centered at $60^{\circ} \mathrm{W}$ and (a) $60^{\circ} \mathrm{N}$, (b) $61^{\circ} \mathrm{N}$, and (c) $62^{\circ} \mathrm{N}$, over 6 days. The final 500-hPa height field, $0000 \mathrm{UTC} 10 \mathrm{March}$ 1965 , is also shown. 
somewhat exaggerated in sharpness because there is no diffusion in the model.

Figure 1 only shows the results for three groups of air parcels. Figure 2 generalizes the results of Fig. 1 and illustrates the chaotic nature of the flow. At 0000 UTC 4 March 1965, a total of 202005 air parcels were uniformly distributed over an area of $25^{\circ} \times 125^{\circ}$ west of Europe. The separation of the parcels was $13.9 \mathrm{~km}$ in a north-south direction. The traveling distance measured in degrees along a great circle between the initial and final location of each parcel was plotted at the initial location. The resulting field was analyzed with an isopleth spacing of $5^{\circ}$ and is shown in Fig. 2a. Where the isopleths are close to each other (high gradient), neighboring parcels will deviate from each other considerably, such as those in group (b) of Fig. 1. There are also large areas where the pathlengths of parcels will not deviate much after 6 days of travel (low gradient). Taking into account that the deviation angle would make the analysis much more complex, only the distance has been measured.

The complexity of the structure in Fig. 2a is repeated at smaller scales. This fine structure is illustrated by zooming, shown with the insets. In Figs. $2 \mathrm{~b}$ and $2 \mathrm{c}$ 40401 parcels have been distributed over $5^{\circ}$ and $0.5^{\circ}$ squares corresponding to a north-south separation of 2.8 and $0.28 \mathrm{~km}$ between the air parcels, respectively.
Two isopleths in Fig. $2 \mathrm{c}$ are labeled. Other isopleths $\left(35^{\circ}-55^{\circ}\right)$ between them are so close to each other that only one line is visible, in spite of strong zooming. Here air parcels initially extremely close to each other show very different final locations after 6 days of travel.

The point groups [(a)-(c)] of Fig. 1 are initially located within Fig. 2b. The middle group, (b), of Fig. 1 is located near the center of Fig. $2 b$ (or $2 c$ ) where the gradient of the field is high. $1^{\circ}$ northward of the center, the gradient in Fig. $2 b$ is very low, and agreeing with this, group (c) of Fig. 1 is still compact after 6 days.

\section{The disturbed case}

The integration scheme will be modified in the disturbed case by adding some averaging, which is a typical element in numerical approximation procedures. For instance, in models applying the Arakawa $\mathrm{C}$ grid, the right-hand-side term in $\partial u / \partial t=f v$ is approximated by averaging the wind values at adjacent grid points.

The horizontal wind in (1) and the associated interpolation formulas are modified by replacing the scalar components $(u, v)$ with

$$
v_{\text {new }}(x, y)=[v(x+a \Delta x, y)+v(x-a \Delta x, y)] / 2
$$

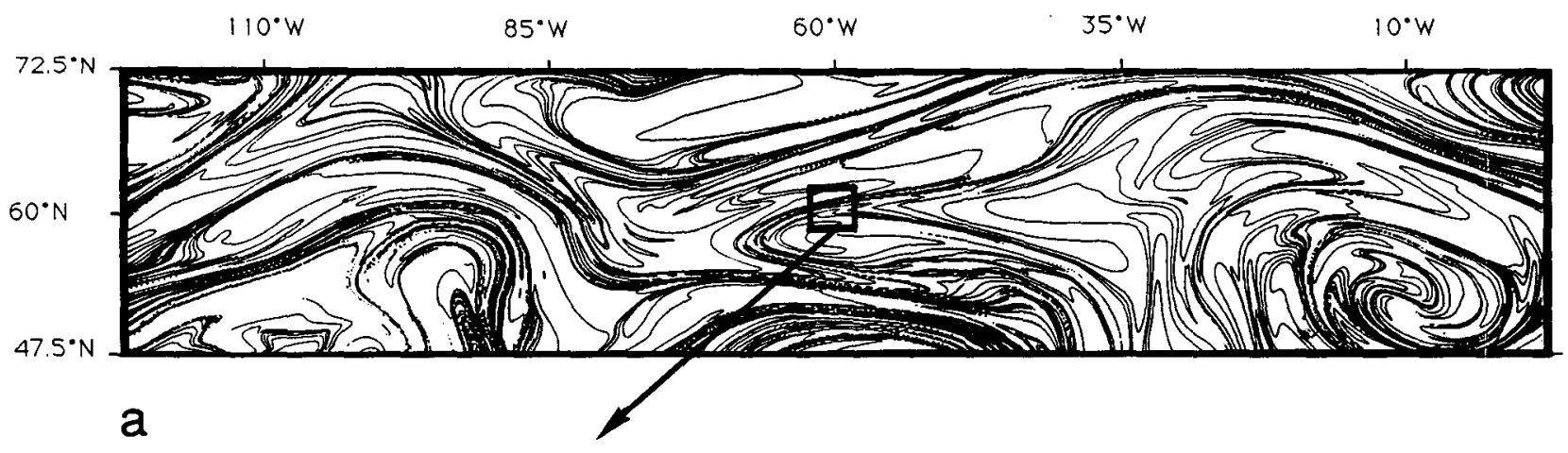

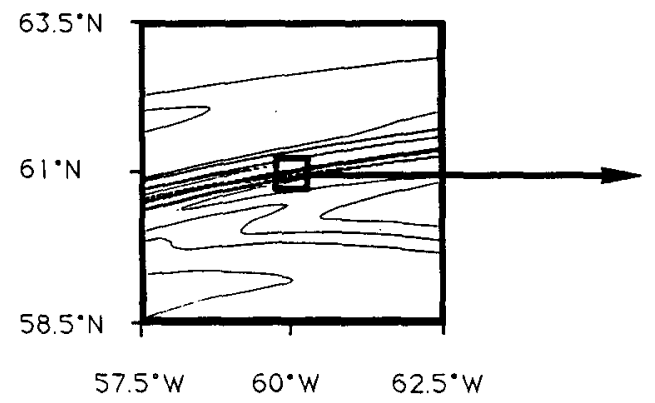

b

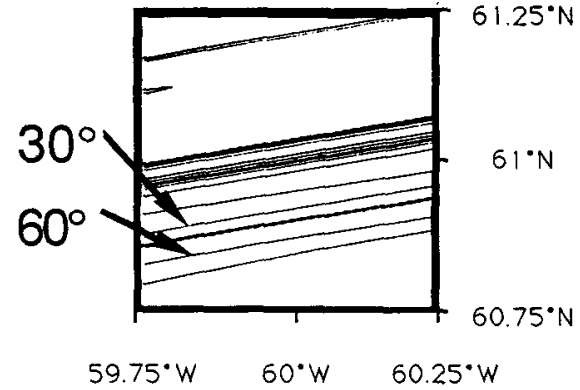

C

FiG. 2. (a) The analyzed field of the distances traveled by 202005 air parcels over 6 days. The fine structure of (a) is illustrated in (b) and (c) by zooming in. In (c), two isopleths of great circle distances $30^{\circ}$ and $60^{\circ}$ are labeled. The spacing of the isopleths is $5^{\circ}$. 
$u_{\text {new }}(x, y)=[u(x, y+a \Delta x)+u(x, y-a \Delta x)] / 2$.

Here $\Delta x$ and $a$ are the grid increment of the original NMC grid and a given coefficient, respectively. The wind values have been interpolated from adjacent points, as described in the Appendix. There is thus no change in these formulas.

When $a=0$ there will be no modification. In the previously presented case of the Arakawa $C$ grid, $a$ $=0.5$. With more complicated numerical approximations, easily $a=1$. Normally, the averaging is applied separately on the terms of a product. Then the effect is cumulative. This corresponds to a large value of $a$.

The qualitative effect (not shown) of the disturbance on Fig. 2 due to (3a) and (3b) is that the complex structure is degenerated, especially when parameter $a$ exceeds 1 . In other words, the most deviating parcels are now kept tighter together, and the chaotic nature of the flow becomes less pronounced.

The effect of the modification due to ( $3 b$ ) at a sharp transition line of $u$, that is, a strong shear in zonal flow, is studied next. Let $u=u_{1}$ and $u=u_{2}$ for $y<0$ and $y$ $>0$, respectively. Thus, adjacent parcels exist traveling at two different speeds. By Eqs. (3b) this is modified to be $u=u_{1}, u=\left(u_{1}+u_{2}\right) / 2$, and $u=u_{2}$ for $y<-a \Delta x$, $-a \Delta x<y<a \Delta x$ and $y>a \Delta x$, respectively. Now a zone of intermediate speeds appears. The air parcels are kept tighter together, and the separation between the two parcel types is less pronounced. The width of the zone is $2 a \Delta x$. Hence, the area (mass) affected is related linearly to the grid increment. Of course the case studied is simplified: a real flow field evolves with time, and confluence of the flow can be more important than the shear.

\section{Comparison of the vorticity transportation in the two cases}

The quantitative effect of the modification in the numerics of the model is studied in the following. The vortex properties of the air parcels, which move into the ridge area shown by $\mathrm{H}$ in Fig. 1, are considered.

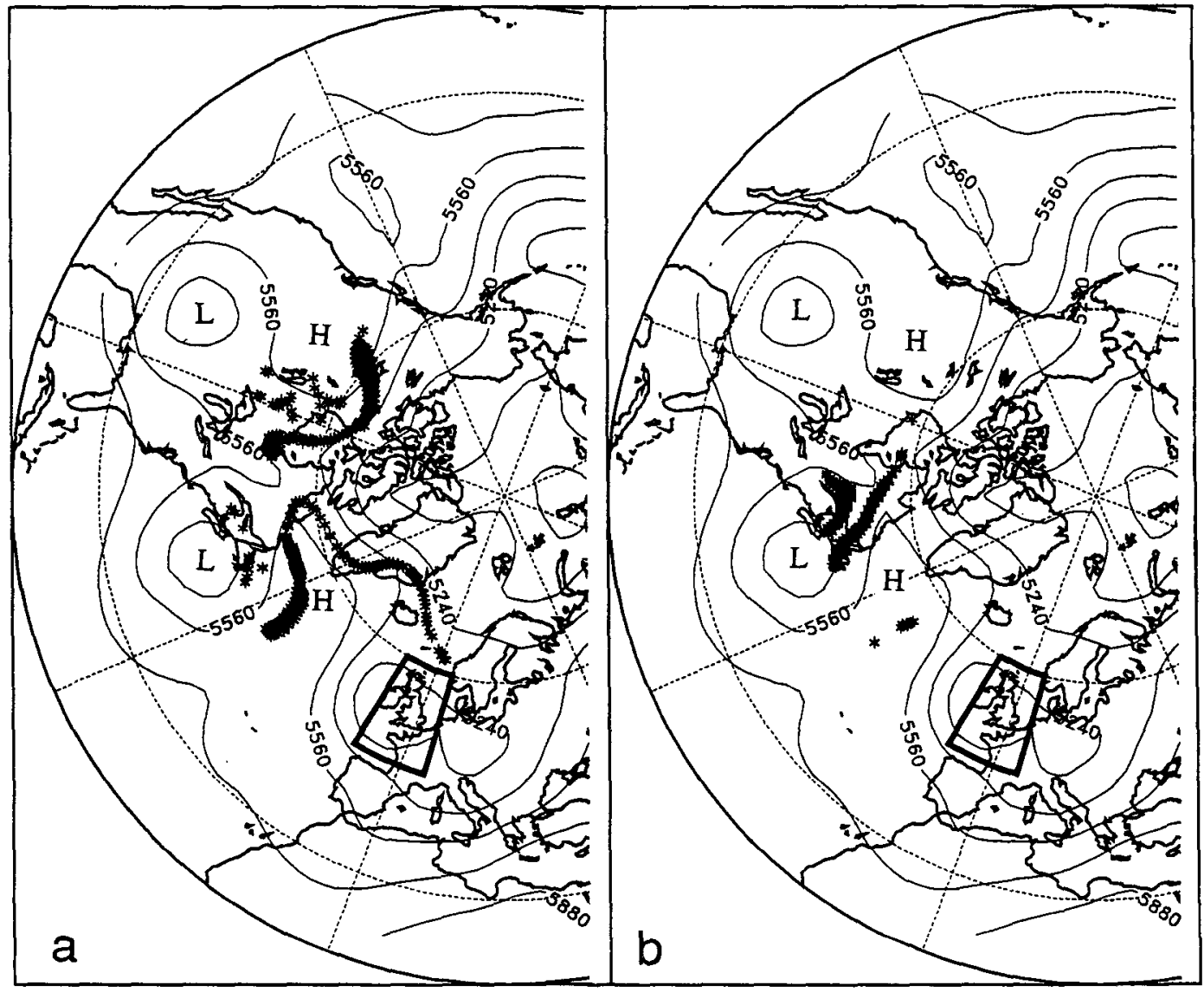

FIG. 3. The initial location (*) of parcels ending up in the ridge (the box shown by a thick line) in the control case (a) with $a=0$ and in the disturbed case (b) with $a=2$. The initial 500-hPa height field is also shown. 
TABLE 1 . The mean relative vorticity $\xi_{\text {final }}$ of parcels ending up in the region of the ridge (cf. Figs. 1 and 3 ) as a function of the averaging parameter $a$.

\begin{tabular}{cccccc}
\hline \hline$a$ & 0 & 0.5 & 1.0 & 1.5 & 2.0 \\
\hline$\xi_{\text {final }}$ & -1.97 & -1.84 & -1.31 & -0.99 & +0.08 \\
\hline
\end{tabular}

The absolute vorticity $\eta$ is given as the sum of the relative vorticity $(\xi)$ and the Coriolis parameter $(f)$. It is assumed that the absolute vorticity of an air parcel is conserved during the transport, that is, $(\xi+f)_{\text {initial }}=(\xi$ $+f)_{\text {final }}$. Thus, $\xi_{\text {final }}=(\xi+f)_{\text {initial }}-f_{\text {final }}$. The mean $\xi_{\text {final }}$ of parcels ending up in the region of the ridge in Fig. 1 (the box outlined in Fig. 3) varies as a function of parameter $a$, as shown in Table 1 .

The disturbance error increases dramatically with $a$. In the control case $(a=0)$ the ridge area is fed by parcels having anticyclonic vorticity. When $a=2$ the vorticity feeding is cyclonic. In this most extreme case, the difference in the initial vorticity is easy to see on weather maps. In Figs. $3 a$ and $3 b$, the initial locations of parcels ending up in the ridge are shown in the control and disturbed cases, respectively. From the height field on 0000 UTC 4 March, it can be concluded that in the two cases the ridge is fed by different parcels having different initial vorticity. In the latter case, the vorticity feeding would prevent any development of the ridge into a blocking high.

Note that the origin of the parcels with $a=2$ is also more southerly than with $a=0$. Thus, the Coriolis term is smaller with $a=2$ (Fig. $3 b$ ). Without this effect the results would be even more pronounced.

"The loss of eddy kinetic energy and the lack of skill in predicting after the early medium range (i.e., 3-4 days) or simulating blocking in climate models remain as outstanding systematic errors" in spite of the encouraging reduction in other systematic errors in recent years (CAS/JSC Working Group on Numerical Experimentation 1988). Tentative explanations of errors in the prediction of blocking highs have been found in the representation of mountains, in horizontal and vertical diffusion, in the parameterization of convection (Tibaldi and Molteni 1990), in the accuracy of the initial conditions (Palmer 1989), and in errors due to resolution in the interaction between ultralong and shorter waves (Tibaldi and Ji 1983).

In the present case, the differences in the parcels streaming into the high area are entirely due to the modifications made in the numerical approximations. These modifications consist of averaging, which is a normal procedure in modeling. Numerical approximations in models can thus lead to erroneous development of highs.

\section{Discussion and conclusions}

In this study we have made use of a coarse grid. This has made the results very dramatic and easy to illustrate. Such numerical conditions are met with in climate models. The routine blocking forecasts are made with global models where the grid increment typically is about one-third of the present one. In the discussion after (3) it was observed that the effect of the erroneous averaging can be related linearly to the grid increment. Accordingly, the error should decrease with increasing resolution (as in Tibaldi and Ji 1983) but not rapidly. This fits with the observation that the skill of forecasting blocking highs has not improved much with better models (CAS / JSC Working Group on Numerical Experimentation 1988; Tibaldi and Molteni 1990).

To feed a blocking pattern with different vorticity requires parcels having a different origin. This is illustrated in Fig. 3 where the parcels reaching the target area originate from different areas. For this to be realized the parcels must have been traveling long enough; that is, the integration period must be sufficiently long. This fits with the observation that forecasting errors in the blocking situations become serious after 3- or 4-day integrations (CAS/JSC Working Group on Numerical Experimentation 1988; Tibaldi and Molteni 1990).

It is obvious that introducing vertical motion and more complicated structures in the model and numerics would increase the error. We have only studied this one randomly chosen case. There are obviously worse cases.

In our approach, any error mechanism deviating the air parcels from their paths in a suitable way can violate

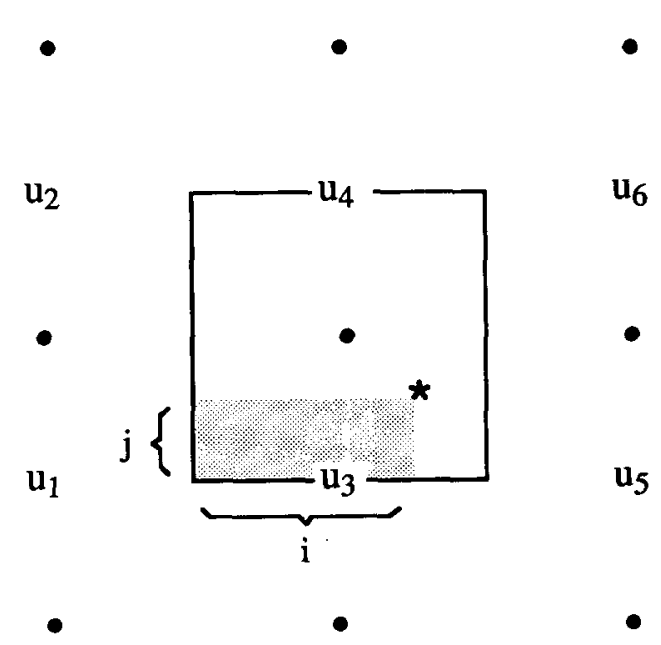

FIG. Al. The nine closest grid points $(\cdot)$ and the $u$ components of the geostrophic wind, denoted by $u_{1}, \ldots, u_{6}$, used in the determination of the $u$ component at the actual point (*). The coordinate of the actual point is $(i, j)$. 
the blocking development. Thus, the results should not be specific for either a Lagrangian model or for certain numerical approximations. Any error source causing similar systematic deviating errors in the movement of chaotically flowing parcels could be serious for blocking development. Our case is only one illustration of such an error.

\section{APPENDIX}

\section{Determination of the Wind at an Arbitrary Point}

First the wind is geostrophically determined at six points from height values at nine adjacent grid points (Fig. A1). A linear interpolation to the actual point is, in the case of the $u$ component, then made as follows:

$$
\begin{gathered}
u(i, j)=\frac{1}{2}\left[u_{1}+u_{3}+j\left(u_{4}-u_{3}\right)+j\left(u_{2}-u_{1}\right)\right] \\
+\frac{i}{2}\left[u_{5}-u_{1}+j\left(u_{6}-u_{5}\right)+j\left(u_{2}-u_{1}\right)\right],
\end{gathered}
$$

where $0 \leqslant i \leqslant 1,0 \leqslant j \leqslant 1$, and $u_{1}, \ldots, u_{6}$, as in Fig. A1.

The $v$ component of the wind is handled in a similar manner.

\section{REFERENCES}

CAS/JSC Working Group on Numerical Experimentation, 1988: Workshop on Systematic Errors in Models of the Atmosphere. Report No. 12. WMO/TD, 273, vii-xvii.

Tibaldi, S., and R. Ji, 1983: On the effect of model resolution on numerical simulation of blocking. Tellus, 35A, 28-38.

- , and F. Molteni, 1990: On the operational predictability of blocking. Tellus, 42A, 343-365.

Palmer, T., 1989: Weather eye on unpredictability. New Sci., 124, $56-59$. 